
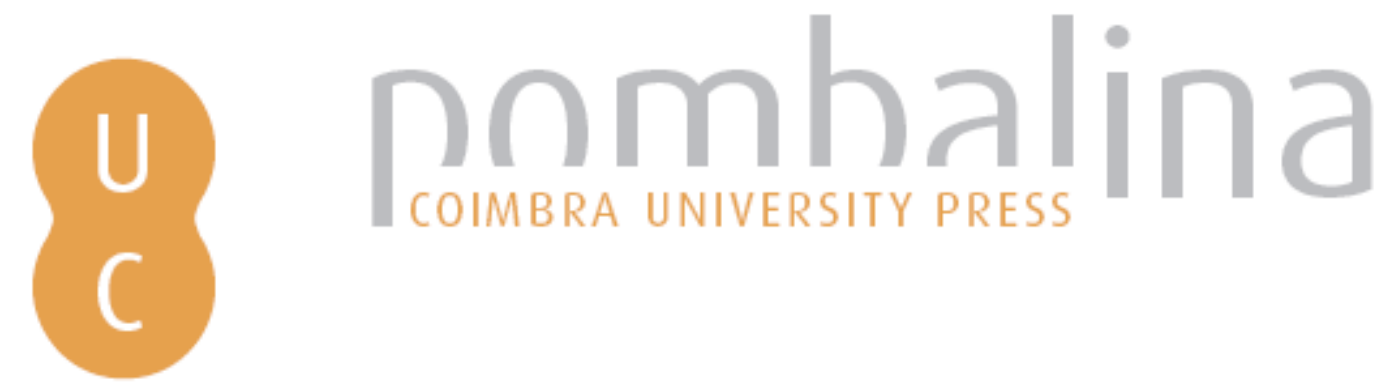

\title{
Cuestiones retórico-traductológicas de los marcadores del discurso (y de su ausencia), ejemplificadas en la traducción española de Atemschaukel (Herta Müller)
}

\author{
Autor(es): $\quad$ Gil, Alberto \\ Publicado por: Imprensa da Universidade de Coimbra \\ URL \\ persistente: URI:http://hdl.handle.net/10316.2/43264 \\ DOI: $\quad$ DOI:https://doi.org/10.14195/978-989-26-1446-5_3 \\ Accessed : $\quad$ 26-Apr-2023 13:36:59
}

A navegação consulta e descarregamento dos títulos inseridos nas Bibliotecas Digitais UC Digitalis, UC Pombalina e UC Impactum, pressupõem a aceitação plena e sem reservas dos Termos e Condições de Uso destas Bibliotecas Digitais, disponíveis em https://digitalis.uc.pt/pt-pt/termos.

Conforme exposto nos referidos Termos e Condições de Uso, o descarregamento de títulos de acesso restrito requer uma licença válida de autorização devendo o utilizador aceder ao(s) documento(s) a partir de um endereço de IP da instituição detentora da supramencionada licença.

Ao utilizador é apenas permitido o descarregamento para uso pessoal, pelo que o emprego do(s) título(s) descarregado(s) para outro fim, designadamente comercial, carece de autorização do respetivo autor ou editor da obra.

Na medida em que todas as obras da UC Digitalis se encontram protegidas pelo Código do Direito de Autor e Direitos Conexos e demais legislação aplicável, toda a cópia, parcial ou total, deste documento, nos casos em que é legalmente admitida, deverá conter ou fazer-se acompanhar por este aviso.

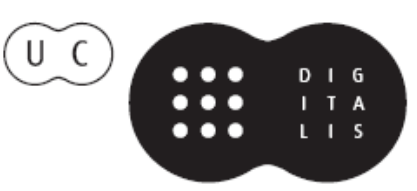


(1) ANA PAULA LOUREIRO GONCEIÇÃO CARAPINHA CORNELIA PLAG

1 (ICOORDS.)

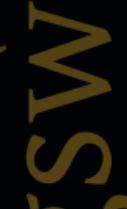

bo

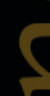

구구요

?ubrigens

(1)

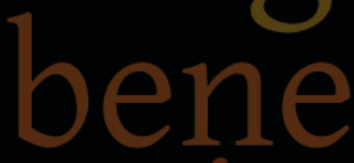

$\bullet$

N

assing

(1)

(2)

MARCADORES

DISCURSIVOS

E(M)

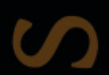

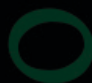

10

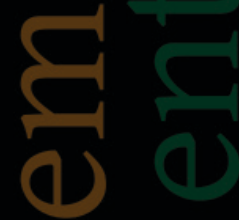

तु

\section{(}

(1)
IMPRENSA DA

UNIVERSIDADE

DE COIMBRA

COIMBRA

UNIVERSITY

PRESS

affettiv 


\title{
CUESTIONES RETÓ㇒ ICO-TRADUCTOLÓGICAS DE LOS MARCADORES DEL DISCURSO ( Y D E S U A US ENCIA), EJ EMPLIFICADA S E N LA T RADUCCIÓN ESPAÑOLA DEATEMSCHAUKEL ( HERTA MÜLLER)
}

\author{
Alberto Gil \\ (Universität des Saarlandes - Saarbrücken) \\ a.gil@mx.uni-saarland.de
}

\begin{abstract}
Los marcadores discursivos (MD) cumplen la importante función de procesar la información. Por eso son un apreciado campo de investigación tanto en la lingüística del texto como en la traductología. Para su estudio importa tanto su presencia como su ausencia, es decir su uso implícito, ya que este último puede transportar instrucciones sutiles e incluso poéticas. En Lingüística se ha tratado esporádicamente esta falta de explicitud en los MD, pero en Traductología no se ha trabajado aún sobre el tema. En el presente artículo se intenta impulsar este estudio poniendo las bases teórico-traductológicas correspondientes y aplicándolas a la traducción española de la sutil y poética novela de Herta Müller, Atemschaukel. En este estudio puede ya entreverse cómo la necesidad de explicitud de ciertos MD en la traducción lleva o bien a la pérdida de matices poéticos o a una objetivación del texto que lo va convirtiendo en casi técnico. Se propone una mayor correspondencia entre hermeneútica y creatividad, observando esta relación bidireccionalmente, es decir, partiendo de que la creatividad que lleva a una mejor comprensión del original es la mejor forma de traducir.
\end{abstract}

Palabras clave: marcadores discursivos; traducción literaria; interpretación; hermenéutica; creatividad 


\begin{abstract}
Discourse markers (DM) play an important role in the processing of information. They are therefore a significant topic of research in Text Linguistics and Translation Studies. Research into not only their presence but their absence is important and in particular their implicit use for conveying subtle and even poetic instructions. This implicit use of DMs has occasionally been the subject of studies in Text Linguistics, but not in Translation. This paper intends to promote this research, establishing initially the theoretical basis of Translation Studies and applying them to the translation into Spanish of Herta Müller's subtle and poetic novel Atemschaukel. In this study, the explicitness of some of the DMs results in the loss of poetic nuance and the objectivation of the translated text turns some parts of the original into rather technical utterances. We propose a translation method in which there is a greater correspondence between hermeneutics and creativity, observing this relationship bidirectionally, in other words, starting with the creativity which leads to a better comprehension of the original.
\end{abstract}

Key words: discourse markers; literary translation; interpretation; hermeneutics; creativity

\title{
Introducción: Marcadores discursivos y traducción
}

Existe consenso en que los marcadores del discurso (en adelante MD) tienen un significado de procesamiento, es decir, que sirven de señalizaciones para ayudar a la interpretación de un texto. Ya Karl Bühler (1982: 390), hablando de los recursos de la anáfora textual, utilizaba la imagen de las flechas, que, si pudieran hablar, irían orientando al lector hacia dónde ha de mirar, para así entablar las relaciones contextuales convenientes. Pero, como no pueden hablar, es el lector quien, con ayuda de estas señales, activa su capacidad cognitiva en la recepción del texto.

En el presente artículo nos preguntamos: Y si faltan estos MD? Bien sea por descuido o bien, más frecuentemente, sobre todo en literatura, porque su falta obedece a una opción estilística. No se 
ha estudiado mucho este tema, si bien se pueden hacer algunas referencias. Catalina Fuentes (2010: 722) señala para estos casos las siguientes posibilidades:

1) El emisor elude su responsabilidad, dejando la comunicación abierta y/o pretendidamente confusa.

2) Se deja al receptor mayor libertad para inferir lo más accesible a su mente.

3) Esta estrategia potencia y enriquece el mensaje, porque admite varias líneas, varias continuaciones de interpretación.

4) En definitiva: es el lector quien tiene que completar lo no expresado explícitamente, interpretarlo.

Esta última actividad hermenéutica es parte constituyente de toda traducción, pero, a su vez, el estudio de las decisiones del traductor (dejar en la traducción, como en el original, el marcador 0 , o bien interpretar ese hueco con un marcador de la lengua de llegada) dará luz, por su explicitud, al estudio de las instrucciones contenidas en los marcadores, al tratar de explicarse por qué se emplean de una manera o de otra.

Así pues, desde esta perspectiva traductológica, nos preguntamos concretamente: ¿Cómo es esta relación entre interpretar y traducir? (En nuestro centro de investigación en Saarbrücken hablamos de la relación entre hermenéutica y creatividad, (cfr. www. www.hermeneutik-und-kreativitaet.de). ¿Se trata de una relación unidireccional o bidireccional? Es decir, ¿solo se puede traducir después de una perfecta hermenéutica o bien es el proceso creativo de la traducción un paso importante para la interpretación, al verse uno obligado a monosemizar lo que en principio era un signo polisémico? La otra cuestión concreta es si en el caso de lo implícito en el original, la explicitud de la traducción sirve para entender mejor el texto o bien desarticula la poeticidad del original, cosificándolo. 
Trataremos de responder a estas preguntas dando los siguientes pasos: en primer lugar, pondremos las bases de una fundamentación teórico-traductológica, para seguidamente pasar a aplicar esos conocimientos a una traducción concreta, en nuestro caso haremos un análisis de pasajes seleccionados de Herta Müller, premio nobel de literatura en 2009, especialmente de su novela Atemschaukel.

\section{Fundamentación teórico-traductológica}

En traductología se ha tratado poco este campo de los MD, pues los estudios de traducciones se concentran sobre todo en fenómenos al parecer de mayor vigencia textual, como vocabulario, macro y microestructuras, cuestiones culturales y de estilo, etc., sin haberse dado mucho cuenta de la relevancia traductológica de estos MD, tan decisivos precisamente para la creación de textualidad desde su perspectiva cognitivo-comunicativa. ${ }^{1}$ De todas formas, se pueden entresacar algunos trabajos al respecto, de gran valor orientador:

a) un estudio básico de Portolés en lengua española del 2002;

b) dos tesis doctorales en lengua alemana que se centran en marcadores específicos: Wienen (2006): los así llamados "conectores clivados" (ej. es por eso que o bien c'est pour cela que) y Schröpf (2009): secuencias conectivas del tipo $y$ además / et en plus o bien y sin embargo / et pourtant.

No conozco estudios sobre el fenómeno de la explicitación de los MD o del mantenimiento de su ausencia en la traducción, si bien, como hemos visto en Catalina Fuentes, se reconoce como cuestión a estudiar. De los estudios mencionados entresacamos las obser-

\footnotetext{
${ }^{1}$ De ahí la importancia de los Colóquios MarDisT de la Universidad de Coimbra.
} 
vaciones que tienen más relación con la cuestión aquí abordada: Portolés (2002) pone de relieve, al comparar las lenguas y estudiar traducciones, que no solo no se puede hablar de una equivalencia de marcadores en las diversas lenguas (155), sino que tampoco existe un paralelismo completo entre las lenguas para procesar la información (156). Precisamente el hecho de que en la lengua de partida no haya un marcador, pero sí se ponga en la de llegada, prueba que no existen instrucciones universales de procesamiento que estén igualmente codificadas en las diferentes lenguas (159).

En las tesis alemanas se profundizan y amplían estas ideas. A la pregunta de por qué no se encuentran frecuentemente equivalentes de marcadores en las traducciones responde Schröpf (2009: 274), como resultado de un análisis amplio de diferentes traducciones y traductores, recalcando que en el original los MD mismos dejan amplios márgenes de interpretación, lo que explica que vengan interpretados diferentemente por los diferentes traductores. Es decir, la polifuncionalidad del marcador lleva consigo una diversidad de interpretaciones y, por consiguiente, de traducciones. Esta pluridimensionalidad de la traducción se acentúa cuando la función principal del marcador consiste en crear presuposiciones, como es el caso de los conectores estudiados por Wienen. En este tipo de MD (cfr. Wienen 2006: 373 y ss.), la traducción está ligada a la reproducción de la estructura de la información, en la que se combinan la perspectiva del emisor (foco-fondo) con la del receptor (tema-rema). En textos como el que analizaremos más abajo en esta contribución, esta función de estructurar la información es de suma importancia, dadas las características propias de la prosa artística.

Resumiendo, podemos constatar que el traducir o no traducir un MD está ligado en primer lugar a la tipología de cada lengua. Además, los márgenes de interpretación pueden variar según los traductores y, finalmente, los MD están muy relacionados con lo implícito, el gran problema de la traducción, pero uno de los más 
interesantes para la investigación, que vale la pena observar un poco más detenidamente.

Ideas de fondo a este respecto, y que conviene discutir, parten no de un traductólogo, sino de un filósofo, de Hans-Georg Gadamer y de sus reflexiones sobre lenguaje y traducción. En su famoso ensayo de 1966 „Mensch und Sprache“ ("El hombre y la lengua”) (1993: 146-154) distingue el conocido hermenéutico tres dimensiones de la traducción, que traemos a colación telegráficamente:

I. el hecho de traducir

II. los límites de la traducción

III. la principal tarea del traductor

ad I. El hecho de traducir

La traducción no es una mera transformación lingüística (Sprachstoff), el traductor es siempre un autor (ein Sagender).

\section{ad II. Los límites de la traducción}

Los límites están en lo implícito. Se traduce lo dicho. El verdadero sentido se despliega en el lenguaje original y se va diluyendo en su repetición y transformación.

\section{ad III. La principal tarea del traductor}

Por eso, el traductor no es un copista del original, sino un escritor que utiliza la lengua para expresar lo que realmente constituye el sentido total del original.

La cuestión de fondo en nuestro caso es si lo implícito es realmente el límite de la traducción o bien el lugar donde se desarrolla la dimensión traductológicamente más importante, pues ahí es donde se cumple la tarea principal del traductor: ser un escritor, pero evidentemente un escritor-transmisor. 
En base a este papel tan activo y creativo del traductor se podría decir que la relación entre hermenéutica y creatividad no es unidireccional, es decir, se ha de entender el texto de origen perfectamente para poder traducirlo, sino que es bidireccional: precisamente en el acto creativo de traducir se llega a comprender definitivamente el texto de origen. No es aquí el lugar para una exposición sistemática de la hermenéutica traductológica, para ello remito a Cercel (2013), que desarrolla el tema tanto histórica como sistemáticamente.

Aquí trataremos de responder a estas preguntas sobre la base de una traducción profesional de un texto de especiales dimensiones poéticas, Atemschaukel, de Herta Müller, preguntándonos si la traducción o no traducción de MD sirve para una interpretación más aguda de las instrucciones contenidas en los marcadores.

\section{Análisis de pasajes seleccionados de Herta Müller, especial- mente de su novela Atemschaukel}

La novela Atemschaukel es un objeto adecuado para el estudio del fenómeno de la presencia o ausencia de marcadores, ya que la prosa de Herta Müller presenta un estilo peculiar, que podríamos llamar "telegráfico-poético". En otro estudio (Gil, 2014) he analizado éste y otros fenómenos de esta prosa como típicos del estilo de Herta Müller. Remito a este trabajo, donde se encuentra abundante bibliografía sobre el leguaje y estilo de la novelista germano-rumana. Aquí nos ceñiremos al aspecto de los MD con el siguiente objetivo: analizar las medidas de compensación para explicitar o no lo que en el estilo del original no se ha hecho patente. Hemos podido observar tres fenómenos de traducción, sobre los que habrá que reflexionar traductológicamente. 
a) Se cambian o se añaden conectores

b) Se utilizan otros recursos, que conllevan sus aciertos y sus problemas

c) No se traducen partículas modales alemanas (modalizadores), con el consiguiente desequilibrio textual

Los dos primeros se ejemplifican con conectores y el tercero con modalizadores. Finalmente trataremos de explicarnos lo que aporta este análisis a la cuestión aquí abordada sobre el procesamiento semántico y su dimensión translatológica.

\section{ad a) Se cambian o se añaden conectores}

(1) Fenómeno: un conector polisémico del original se monosemantiza en la traducción:

\begin{tabular}{|l|ll|}
\hline Am 9. Mai jährte sich zum ersten & El 9 de mayo se cumplió el primer
\end{tabular} Mal der Frieden. Und er hat uns aniversario de la paz. Mas tampoco wieder nichts genutzt, für uns nos sirvió de nada, fue nuestro segunwar es das zweite Lagerjahr. (127) do año en el campo de trabajo. (115)

En español sería también posible utilizar el conector $\mathbf{Y}$ con su polisemia aditiva y adversativa. El traductor opta aquí, sin embargo, por la explicitación de la relación semántica adversativa, lo que, sin embargo, no refleja la sensación de desilusión del und alemán combinado con el marcador aspectual repetitivo wieder. En español se reflejaría con Y volvió a no servirnos de nada.

El ejemplo (2) constituye un caso claro de interpretación: al conector 0 del original se le añade la conjunción final para que: 
\begin{tabular}{|l|l|}
\hline Unsere Fuhren wurden auch nicht & Tampoco nosotros la transportába-
\end{tabular} wegen der Schönheit gemacht. Wir mos por su belleza. Traíamos holten tonnenweise gelben Sand, toneladas de arena amarilla para que die Baustellen fraßen ihn. (127) las obras la devorasen. (115 y s.)

Nos preguntamos: ¿Qué consecuencias tiene este acto de creatividad? En alemán, el narrador es, sobre la base de la concatenación asindética (Wir holten tonnenweise gelben Sand, die Baustellen fraßen ihn), un mero observador de hechos, "no piensa", constata. En español, con la conjunción para que, el narrador aporta implícitamente su reflexión sobre el sentido o sinsentido del trabajo que están haciendo, al explicitar la causa final de su trabajo (Traíamos toneladas de arena amarilla para que...).

En el ejemplo (3) sigue a una comparación irreal una afirmación categórica, reforzada por el orden marcado de las palabras con la estructura Rema-Tema:

(3) (contexto: en una pausa del trabajo, los dos prisioneros se dejan caer en la arena)

\begin{tabular}{|l|l|}
\hline Es kam Ferne in den Kopf, als & La lejanía se adentraba en tu cabeza, \\
wäre man abgehauen und gehöre & como si te hubieras largado y formases \\
jedem Sand in jeder Gegend der & parte de la arena de cualquier lugar del \\
Welt, nicht der Zwangsarbeit & mundo y no de los trabajos forzados \\
hier. Flucht im Liegen war das. & $\begin{array}{l}\text { de aquí. Era como huir tumbado. (116 } \\
\text { (129) }\end{array}$ \\
\hline
\end{tabular}

La cuestión planteada por Schröpf de que diferentes traductores interpretarían diferentemente las instrucciones de los marcadores se pone claramente de relieve en este ejemplo. Constatamos que la traducción española opta por el paralelismo lógico: expresión de dos irrealidades en el pensamiento del narrador: (1) (era) como si te hubieras largado y (2) Era como huir tumbado. Se pone de ma- 
nifiesto una estructura cuidada del texto. En el original podemos observar un orden marcado en el que se focaliza el rema al principio de la última frase, expresando así más claramente la emocionalidad espontánea, sin progresión temática, del hablante. Dado que el español no goza de esa libertad de orden de las palabras, se podría echar mano de una estructura clivada como no era sino huir tumbado, que focaliza también el rema. En este "desorden" sintáctico se expresa mejor una especie de reproducción más directa de los pensamientos espontáneos del narrador.

ad b) Se utilizan otros recursos, que conllevan sus aciertos y sus problemas

Un fenómeno relativamente corriente en traducción es la conciencia del contraste de dos sistemas lingüísticos diferentes, estudiando las posibilidades de la lengua de llegada para llegar a una equivalencia o semiequivalencia. Este fenómeno se da en el ejemplo (4):

\begin{tabular}{|l|l|}
\hline Wir sind zu zweit, der Albert Gion & Albert Gion y yo somos dos trabajado-
\end{tabular} und ich, zwei Kellerleute unter den res del sótano situado debajo de las Dampfkesseln der Fabrik. (168) calderas de vapor de la fábrica. (151)

La aposición es estructuralmente posible en las dos lenguas. El texto español, que no ha optado por ella, da la impresión de haber sido reformulado en la mente de la traductora, prescindiendo de la aposición y con ella del pequeño suspense del original: somos dos (wir sind zu zweit), añadiendo luego los nombres. Con esta especie de "objetivación" se resuelve también el problema estructural: como el sintagma alemán $\mathrm{N}+$ Prep.+N no se suele utilizar en español, excepto en caso de complemento determinativo, la traductora añade situado, que junto con la traducción literal del 
compuesto alemán Dampfkessel por calderas de vapor produce una repetición triple de de, que destruye el ritmo dactílico del sintagma alemán (zwei Kellerleute unter den Dampfkesseln der Fabrik). Traductológicamente se puede decir que la hermenéutica (que aquí ha funcionado bien) no es suficiente para una traducción adecuada, ya que - sobre todo en textos con vocación poética - falta la creatividad de recrear matices informativo-estructurales y poéticos.

Sin embargo, cuando la estructura es idéntica en ambas lenguas, el traductor puede llegar a verdaderos pasajes poéticos, como es el caso de (5):

\begin{tabular}{|ll|l|}
\hline In der Hautundknochenzeit hatte & En la época de piel y huesos yo no
\end{tabular} ich nichts mehr im Hirn außer dem tenía otra cosa en la cabeza que la ewigen sirrenden Leierkasten, der eterna cantinela machacona que Tag und Nacht wiederholte: Kälte repetía día y noche: el frío corta, el schneidet, Hunger betrügt, Müdigkeit hambre engaña, el cansancio pesa, lastet, Heimweh zehrt, Wanzen und la nostalgia consume, los chinches Läuse beißen. (249)

y piojos pican. (225)

El MD de los dos puntos es en ambas lenguas un marcador introductor de una enumeración o de citas textuales. En la traducción española - como pide la gramática - se añade el artículo, pero se mantiene la bimembración y al final la trimembración. Además, se consigue un efecto poético con las aliteraciones de la enumeración (piojos pican). Dicho sea solo de paso, el compuesto creativo Hautundknochenzeit traducido como época de piel y huesos no tiene mucho sentido. En alemán, la autora ha sido creativa, jugando con la asociación con la expresión popular: Sauregurkenzeit (= tiempos difíciles); en español se podría decir creativamente: el-tiempo-enque-te-quedas-en-los-huesos)

Wienen (cfr. arriba) llama la atención sobre la estrecha relación entre los MD (y su traducción) con fenómenos de la estructura de 
la información. En el ejemplo (6) se mantiene el marcador Aber/ Pero, rompiéndose, sin embargo, la textura (context boundness) del original con un salto temático. La razón parece ser también aquí (cfr. ej. 4) querer organizar la frase según estructuras lógicas (setting, sujeto, verbo, objeto):

\begin{tabular}{|l|l|}
\hline Statt auf solche Gedanken zu kom- & En lugar de entretenerme con estas \\
men, könnte ich etwas lesen. Aber & ocurrencias, podría leer algo. Pero \\
den schrecklichen Zarathustra, den & hace tiempo que vendí como pa- \\
dicken Faust und den dünnge- & pel de fumar, para calmar un poco \\
druckten Weinheber habe ich für & el hambre, el terrible Zaratustra, el \\
ein bisschen Hungerstille längst als & grueso Fausto y el Weinheber impre- \\
Zigarettenpapier verkauft. (205) & so en papel de biblia. (184) \\
\hline
\end{tabular}

En alemán se consigue el enlace textual (context boundness) con la enumeración de los títulos después del verbo lesen (leer): Zarathustra, Faust, etc.; en español se ordena la frase (hace tiempo que vendi como papel de fumar, etc.) y al final se enumeran los títulos. Se puede decir que la traducción del marcador con el mismo valor semántico no es suficiente. MD y orden de las palabras forman una unidad como expresión de un mensaje con toda su fuerza comunicativa y poética.

\section{ad c) No se traducen partículas modales alemanas (modali- zadores), con el consiguiente desequilibrio textual}

Ya desde hace tiempo (cfr. Gil/Schmitt 1996: 310 y ss.) es conocido el problema de la traducción de marcadores, cuando no existen en la lengua de llegada. En la relación alemán-español se refleja este fenómeno en el tema de las partículas modales.

En (7) propongo un ejemplo que parece reforzar la ya citada tesis de Gadamer sobre los límites de la traducción, es decir, cuando se 
ve la necesidad de reproducir lo implícito. En alemán cumplen este cometido las así llamadas partículas modales, usadas fundamentalmente en diálogos, con funciones determinadas.

(7) (contexto: un encuentro con el temido vigilante Tur Prikulitsch)

\begin{tabular}{|c|c|}
\hline $\begin{array}{l}\text { Wie ist es denn bei euch im Keller. } \\
\text { Gemütlich, sagte ich, jede Schicht } \\
\text { ist ein Kunstwerk. } \\
\text { (...) } \\
\text { Wie dreckig gestern Dein Gesicht } \\
\text { war, sagte er, und wie aus allen } \\
\text { Löchern Deiner Kappe die Därme } \\
\text { hingen. } \\
\text { Mach ja nichts, sagte ich, } \\
\text { Kohlestaub ist pelzig und finger- } \\
\text { dick. (169) }\end{array}$ & $\begin{array}{l}\text { Qué tal os va en el sótano } \\
\text { Tan ricamente, contesté, cada turno es } \\
\text { una obra de arte. } \\
\text { (...) } \\
\text { Qué sucia tenías la cara ayer, exclamó, } \\
\text { y cómo te asomaban las entrañas por } \\
\text { todos los agujeros de la gorra. } \\
\text { No importa, repuse, el polvo del car- } \\
\text { bón es afelpado y tiene un dedo de } \\
\text { grueso. (152) }\end{array}$ \\
\hline
\end{tabular}

En el contexto de la novela, denn finge un tono amable conversacional, una toma de contacto; mientras que ja tiene una función reforzativa, como un adverbio focalizador, delante de nichts, en el sentido de "absolutamente nada". Ambas partículas revelan un estado de ánimo que finge naturalidad, pero que en el primer caso produce desconfianza y en el segundo miedo a que le pase algo peor si se queja.

En la traducción española se opta por los recursos de variedad léxica, como el coloquial Qué tal os va (...). Tan ricamente.. O bien la expresión categórica, no modalizada, No importa. Un esfuerzo creativo ayudaría a encontrar un MD español como $\boldsymbol{Y}$ qué tal os va en el sótano para el primer caso, con el que se imitaría mejor el fingimiento de inocente conversación iniciada por el temido vigilante. En el segundo caso, se podría pensar en la expresión $\boldsymbol{Y}$ qué mas $d a$, con la que se expresaría el esfuerzo del protagonista por quitar importancia al asunto. 
Cabe pues una argumentación diferente a la de Gadamer, mostrando que los límites de la traducción no son la implicitud, sino que ésta es el desafío más interesante para la creatividad del traductor.

\section{Conclusión}

¿Que podemos entresacar del análisis efectuado? A nivel comunicativo-subcutáneo, la presente traducción parece quedarse en los límites de que habla Gadamer, es decir, concentrarse en lo explícitamente expresado. Con ello, el texto adquiere un matiz más objetivo y funcional perdiendo en nivel poético.

Respecto a la pregunta de fondo de cómo se conjugan las dimensiones de hermenéutica y creatividad, se puede deducir del presente análisis que, por un lado, la creatividad no siempre es positiva, sobre todo si se reelabora el texto haciéndolo más explícito, más gramaticalmente lógico a costa de su poeticidad. El secreto parece estar entre líneas, es decir, en el amor del traductor por el detalle, que primero con competencia lingüística y cultural ha sido captado hermenéuticamente en el original, para luego buscar los recursos más apropiados en la lengua de llegada.

El presente trabajo es un pequeño esbozo, pero que pretende abrir algunas perspectivas para la investigación traductológica posterior: Partiendo de la base de que los MD son procesadores de información, se podrían añadir investigaciones traductológico-estilísticas en torno a la solución de los problemas que plantean estos elementos lingüísticos preguntándose si se puede reconocer en un traductor un estilo propio, entre otras cosas, porque da soluciones regulares a problemas similares o bien se concentra en resolver las cuestiones por separado, es decir, sin un concepto traductológico previo. También cabe preguntarse: ¿Es este concepto, en que se unen hermenéutica y creatividad, una medida para definir la calidad 
de un traductor? Finalmente, estudiando las soluciones creativas adoptadas por el traductor, se puede hacer la pregunta inversa: ¿Qué repercusiones puede tener la creatividad traductora para la comprensión del original?

\section{Referencias}

\section{A) Corpus}

Müller, Herta (2009). Atemschaukel. München: Carl Hanser.

Müller, Herta (2011). Todo lo que tengo lo llevo conmigo. Traducción: Rosa Pilar Blanco. Madrid: Santillana.

\section{B) Estudios}

Bühler, Karl (1982). Sprachtheorie. Die Darstellungsfunktion der Sprache. Stuttgart, New York: Gustav Fischer.

Cercel, Larisa (2013). Übersetzungshermeneutik. Historische und systematische Grundlegung. St. Ingbert: Röhrig Universitätsverlag (= Hermeneutik und Kreativität Bd. 1).

Fuentes Rodríguez, Catalina (2010). Los marcadores del discurso y la lingüística aplicada. In: Loureda Lamas, Óscar/Acín Villa, Esperanza (Coords.), Los estudios sobre marcadores del discurso en español, hoy, Madrid: Arco/Libros, 689-746.

Gadamer, Hans-Georg (1966). Mensch und Sprache. In ders. Hermeneutik II. Gesammelte Werke 2, Tübingen: J. C. B. Mohr 1993, 146-154.

Gil, Alberto / Schmitt, Christian (Eds.) (1996), Kohäsion, Kohärenz, Modalität in Texten romanischer Sprachen. Bonn: Romanistischer Verlag.

Gil, Alberto (2014). Kreativität und Problemlöseverfahren als translatologische Größen, am Beispiel der spanischen Übersetzung von Herta Müllers Atemschaukel. In Kunz, Kerstin / Teich, Elke / Hansen-Schirra, Silvia / Neumann, Stella / Daut, Peggy (Eds.), Caught in the Middle - Language Use and Translation. Festschrift for Erich Steiner on the Occasion of his 60th Birthday. Saarbrücken: Universität des Saarlandes universaar, 129 - 145.

Portolés Lázaro, José (2002). Marcadores del discurso y traducción. In: Fuentes Morán, María Teresa / García Palacios, Joaquín (Eds.), Texto, terminología y traducción. Salamanca: Almar, 145-167.

Schröpf, Ramona (2009). Translatorische Dimensionen von Konnektorensequenzen im Spanischen und Französischen. Ein Beitrag zur linguistisch orientierten Übersetzungswissenschaft Romanisch-Deutsch. Frankfurt a.M. etc.: Peter Lang.

Wienen, Ursula (2006). Zur Übersetzbarkeit markierter Kohäsionsformen. Eine funktionale Studie zum Kontinuum von Spaltadverbialen und Spaltkonnektoren im Spanischen, Französischen und Deutschen. Frankfurt a.M. etc.: Peter Lang. 